\title{
COMPARISON OF A NEW EQUATION DESCRIBING FATIGUE CRACK GROWTH CURVES WITH THE NASGRO EQUATION
}

The so-called NASGRO equation allows a very good description of fatigue crack growth curves, i.e. the dependence of crack length increase per one fatigue cycle da/dN on the stress intensity factor range $\Delta K$. In 1999 the first author of this paper published quite similar equation, which written for given loading cycle asymmetry with positive stress ratio $R$ contains the same parameters having similar meaning as in the NASGRO equation. In most cases studied by the authors this equation leads to a better fit than the NASGRO equation, above all when the experimental curve contains relatively long Paris straight line and/or relatively sharp bend from this line to the threshold stress intensity factor range. The generalization of the NASGRO equation for various values of stress ratio $R$ was made in a quite complicated way. The new equation was generalized using the Walker model based on the relation $\Delta K(R)=\Delta K(0)(1-R)^{-m}$ which is valid also for threshold value $\Delta K_{\text {th }}$ but not for critical stress intensity $K_{c}$ being a constant independent of $R$. Then the shift of the growth curves with the change of $R$ ratio is described only by one parameter $m(0<m<1)$ both for positive and for negative values of $R$. It means that the crack closure models are very important for explanation and deep study of fatigue crack growth mainly in negative $R$ region but play no crucial role in a phenomenological description of the growth in the case of short-term tests in non-aggressive media.

\section{Introduction}

The NASGRO is a commercial complex of very sophisticated and deeply developed computer programs covering all necessary procedures dealing with the growth of long fatigue cracks, including extensive database of experimentally determined fatigue crack growth curves for various structural materials. Some of the parts of the NASGRO program are freely accessible as a demoversion including reference manual [1]. Here so-called NASGRO equation can be found, which describes fatigue crack growth curves, i.e. the dependence of crack length increase per one fatigue cycle $d a / d N$ on stress intensity factor range $\Delta K$. For special case of repeating tension (i.e. for stress intensity parameter $R=0$ ) it can be written in the form

$$
\frac{d a}{d N}(\Delta K)=C \Delta K^{n} \frac{\left(1-\frac{\Delta K_{t h}}{\Delta K}\right)^{p}}{\left(1-\frac{\Delta K}{K_{c}}\right)^{q}}
$$

It contains six parameters: $C$ and $\mathrm{n}$ are connected with the Paris straight line, $\Delta K_{t h}$ is threshold stress intensity factor range, $K_{c}$ is critical stress intensity, and $p, q$ are exponents describing the bends of the curve from the Paris straight line to $\Delta K_{t h}$ and $K_{c}$, respectively.
In 1999 one of authors of this contribution published an equation for the description of fatigue crack growth curves [2] which can be rewritten into the form

$$
\frac{d a}{d N}(\Delta K)=C \Delta K^{n} \frac{1-\left(\frac{\Delta K_{t h}}{\Delta K}\right)^{p}}{1-\left(\frac{\Delta K}{K_{c}}\right)^{q}} .
$$

This form is very similar to the NASGRO equation and includes the same parameters having nearly the same meaning as in the NASGRO equation. Both equations describe all three regions of a fatigue crack growth curve: region I - initiation, region II stable propagation, and region III - unstable fracture. The equations (1) and (2) are written in very useful forms in which the description of single regions is separated: the term before the fraction describes the Paris straight line (if the log-log fit is considered) typical for the stable propagation region, the numerator of the fraction describes the bend of a curve in the initiation region to the threshold stress intensity factor range, and the denominator of the fraction describes the region of unstable fracture where the growth rate increases over all limits. These special forms of the equations can be used as a building kit, because they allow simple adjustment for the cases when only two of all three regions are covered with experimental results and thus only the terms corresponding to the mentioned regions are included into the equations. E.g., the equations only for regions II and III are

\footnotetext{
* Jan Kohout ${ }^{1}$, Stanislav Věchet ${ }^{2}$, Vojtěch Hrubý ${ }^{3}$

${ }^{1}$ Department of Mathematics and Physics, Military Technology Faculty, University of Defence, 61200 Brno, Czech Republic, E-mail:

Jan.Kohout@unob.cz

${ }^{2}$ Institute of Materials Science and Engineering, Faculty of Mechanical Engineering, Brno University of Technology, 61669 Brno, Czech Republic

${ }^{3}$ Department of Mechanical Engineering, Military Technology Faculty, University of Defence, 61200 Brno, Czech Republic
} 


$$
\frac{d a}{d N}(\Delta K)=C \Delta K^{n} \frac{1}{\left(1-\frac{\Delta K}{K_{c}}\right)^{q}} \text { or } \frac{d a}{d N}(\Delta K)=C \Delta K^{n} \frac{1}{1-\left(\frac{\Delta K}{K_{c}}\right)^{q}}
$$

For various values of parameter $R$ the NASGRO equation is written in the form [1]

$$
\frac{d a}{d N}(\Delta K, R)=C\left[\frac{1-f(R)}{1-R}\right]^{n} \Delta K^{n} \frac{\left(1-\frac{\Delta K_{t h}}{\Delta K}\right)^{p}}{\left[1-\frac{\Delta K}{K_{c}(1-R)}\right]^{q}},
$$

where $f(R)=K_{o p} / K_{\max }$ is crack opening function generally expressed by a polynomial of $R$ with rather complicated coefficients. Only in special cases it is the linear function of $R$ or directly equals to $R$ (see [1]). Generalization of Eq. (2) was based on the Walker approach [3]. Walker introduced so-called effective value of stress intensity factor range $\Delta \bar{K}$ given by the relation

$$
\Delta \bar{K}=K_{\max }(1-R)^{\gamma}=\Delta K(1-R)^{\gamma-1}
$$

from which the stress intensity factor range $\Delta K$ for various values of parameter $R$ can be recalculated according to the relation

$$
\Delta K(R)=\Delta K(0)(1-R)^{\gamma-1}=\Delta K(0)(1-R)^{m} .
$$

The exponents $\gamma$ and $m$ used in the recalculations can gain values between 0 and 1 . The generalization of Eq. (2) for various loading cycle asymmetry can be made replacing $\Delta K=\Delta K(0)$ by the term $\Delta K(R)=\Delta K(1-R)^{-m}$ with the exception of the denominator of the fraction where $\Delta K$ must be replaced by $K_{\max }=$ $=\Delta K /(1-R)$ similarly to the generalization of the NASGRO equation (unstable fracture appears when the maximum stress intensity $K_{\max }$ increases close to the critical stress intensity $K_{c}$ ). The threshold stress intensity factor range $\Delta K_{t h 0}=\Delta K_{t h}(0)$ for $R=0$ and critical stress intensity Kc are independent of $R$ and therefore they remain without any change. Thus the following equation

$$
\frac{d a}{d N}(\Delta K, R)=C\left[\frac{\Delta K}{(1-R)^{m}}\right]^{n} \frac{1-\left[\frac{\Delta K_{t h 0}(1-R)^{m}}{\Delta K}\right]^{p}}{1-\left[\frac{\Delta K}{K_{c}(1-R)}\right]^{q}}
$$

was obtained, see [2]. Considering the rather complicated expression of $f(R)$ function, this equation is considerably simpler than the corresponding NASGRO equation (4). On the other hand, Eq. (4) could have a little wider ability to fit various courses of fatigue crack growth curves.

The curves of fatigue crack growth are usually plotted in a log$\log$ coordinates in which the Paris-Erdogan equation is presented as a straight line. In one curve the crack growth rate changes by many orders (up to $10^{6}$ times or even more) and it is usually determined with approximately constant variation (i.e. relative uncer- tainty). For this reason it is better to use not crack growth rate but its logarithm in regression.

\section{Comparison of both equations for given loading cycle asymmetry}

Eqs (1) and (2) should be compared with respect to their ability to fit real experimentally measured fatigue crack growth curves. For comparison of Eqs (1) and (2) the curve published in [1] (Fig. 14 on p. 27) was used. The results of regression are presented in Fig. 1. Both regression curves are nearly indistinguishable in the interval of stress intensity factor range covered by the results of experimental measurements. The sum of squares of deviations for the NASGRO equation is higher by $5.3 \%$ than for the new equation. It means that Eq. (2) is more suitable for regression but in fact this difference is not conclusive. A more substantial difference between both curves appears to be above the interval of the stress intensity factor range covered by the experimental results where the curves substantially diverge. This divergence can be presented by the values of critical stress intensity $K_{c}$ determined by regression, i.e. $(75.8 \pm 48.6) \mathrm{MPa} \mathrm{m}^{1 / 2}$ and $(60.2 \pm 6.2) \mathrm{MPa} \mathrm{m}^{1 / 2}$ using Eq. (1) and Eq. (2), respectively. The standard deviations of $K_{c}$ values differ by an order, which is a very conclusive argument. It can be concluded that the extrapolation behaviour of the new equation is substantially better than that one of the NASGRO equation.

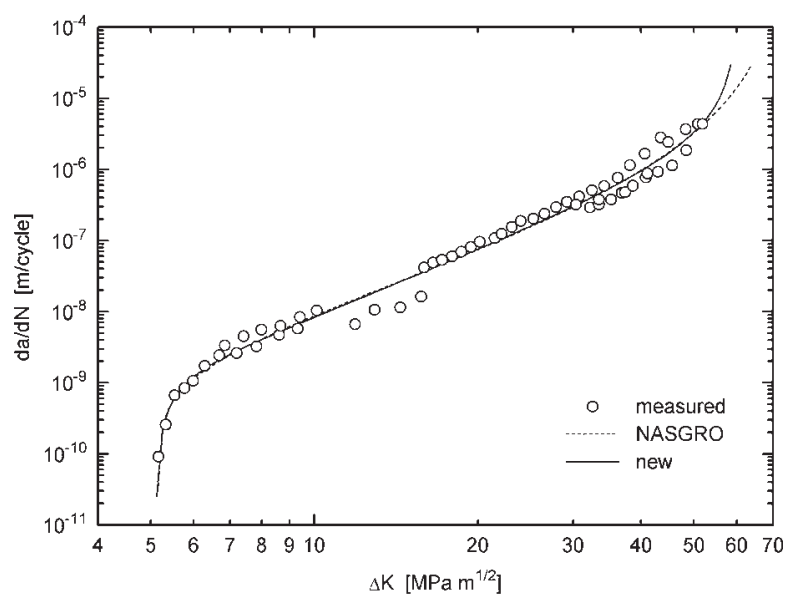

Fig. 1 Fatigue crack growth curve from [1] fitted using Eqs (1) and (2)

\section{New equation for various loading cycle asymmetries}

Direct comparison of Eqs (4) and (7) describing the fatigue crack growth curves for different loading cycle asymmetries is dif- 
ficult just to the complexity of $f(R)$ function. Therefore, only the ability of Eq. (7) to fit the curves for different $R$ will be verified. For this reason, the curves for the values of loading cycle asymmetry parameter $R=0.1$ and 0.5 published by Forman and $\mathrm{Hu}$ [4] were used. The result of regression is presented in Fig. 2. It can be seen that the experimental points are successfully fitted by the curves and their shift can be described by the only parameter $m=(0.52 \pm 0.03)$.

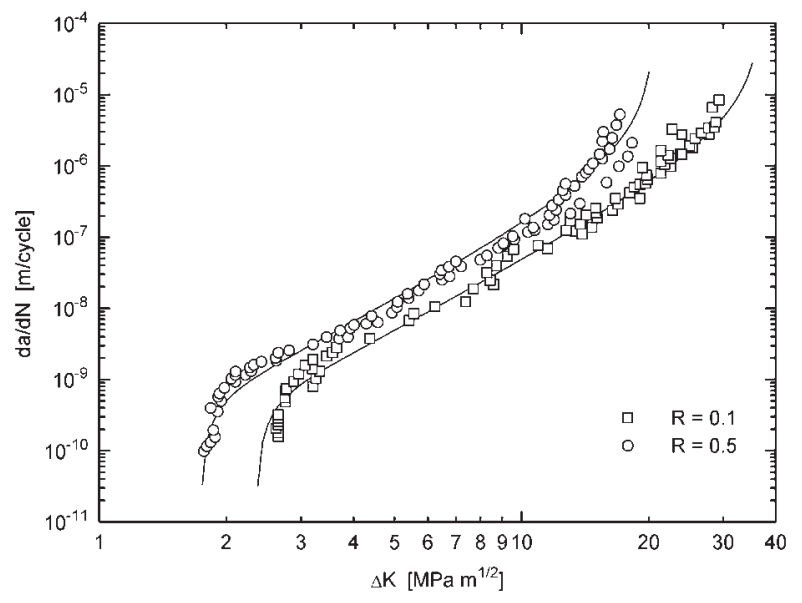

Fig. 2 Fatigue crack growth curves for different $R$ values from [4] fitted using Eq. (7)

Validity of Eq. (7) can be questionable mainly in the case of negative values of loading cycle asymmetry parameter $R$ when the closure of fatigue crack plays a crucial role. Fig. 3, presenting fatigue crack growth curves determined for values $R=0,-0.43,-1$, and -2.33 by Klesnil and Lukáš [5, 6], however shows that the Walker approach expressed by Eq. (6) is very well applicable also for negative $R$ values. For regression shown in Fig. 3 Eq. (7) was used in its simplified form

$$
\frac{d a}{d N}(\Delta K, R)=C\left\{\left[\frac{\Delta K}{(1-R)^{m}}\right]^{n}-\Delta K_{t h 0}^{n}\right\}
$$

which can be obtained from Eq. (7) using following simplifications: (i) the region III of unstable fraction is not considered (denominator in Eq. (7) is not used) and (ii) the equality $n=p$ is considered. For $R=0$ Eq. (8) is identical with the Klesnil-Lukás equation [7], which was originally derived for $R=-1$ and the stress intensity factor amplitude $K_{a}$. The fit based on Eq. (8) is presented in Fig. 3 by the dashed lines.

\section{Exchange of variables in regression}

The fatigue crack growth curve covering the whole interval of stress intensity factor range has two asymptotes $\Delta K=\Delta K_{t h}(R)$ and $\Delta K=K_{c}(1-R)$. The consequence of these vertical asymptotes is that $\Delta K_{t h}(R)$ must be less than all values of $\Delta K$ and $K_{c}(1-R)$ has to be greater than all values of $\Delta K$. It means that, e.g., the minimum value of $\Delta K$ has unacceptably strong influence on $\Delta K_{t h}(R)$ while the influence of all others can be negligible. This unpleasant feature of the $d a / d N=f(\Delta K)$ regression can be avoided by the exchange of variables leading to the $\Delta K=f^{-1}(d a / d N)$ regression. In the case of Eq. (8) the inverse function can be simply expressed in the form

$$
\Delta K\left(\frac{d a}{d N}, R\right)=\left(1-R^{) m}\left[\frac{1}{C} \frac{d a}{d N}+\Delta K_{t h 0}^{n}\right]^{1 / n} .\right.
$$

The fit based on Eq. (9) is presented in Fig. 3 by the full lines.

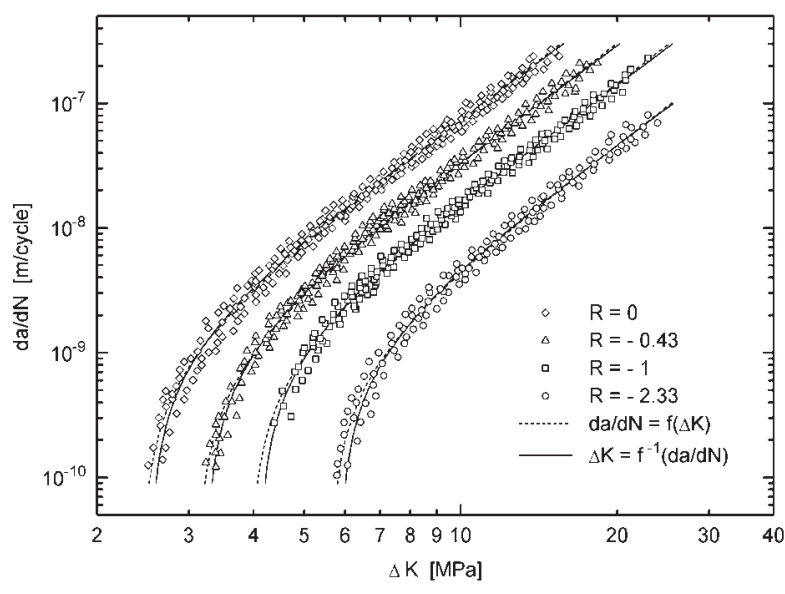

Fig. 3 Fatigue crack growth curves for different $R$ values from [5, 6] fitted using Eqs (8) and (9)

Generally, however the inverse function is not always possible to express explicitly (see, e.g., Eqs (1), (2), (4), and (7)). Then, the inverse function should be calculated numerically using, e.g., the Newton method or the regula falsi method which can be simply implemented into a regression procedure. Unfortunately, some of the simple regression procedures use only explicitly expressible regression functions as a standard and implicitly given functions cannot be used or substantial complications are connected with their application. The comparison of regressions using the regression function $d a / d N=f(\Delta K)$, see Eq. (7), and corresponding inverse function $\Delta K=f^{-1}(d a / d N)$ is shown in Fig. 4 for the same set of experimental data as shown in Fig. 1. The regula falsi method was used for numerical calculation of inverse function because in comparison with the Newton method the derivative of the primary function is not required. Again, as the values of the stress intensity factor range change by more than one order and they are usually determined with an approximately constant variation, the logarithm of $\Delta K$ should be used in regression.

The difference between the curves in Fig. 4 is evident near the asymptotes, especially in the region of unstable fracture. From the point of view of regression the $\Delta K=f^{-1}(d a / d N)$ fit is a little better (so-called corrected index of determination is equal to 0.9854 in this case compared with 0.9843 for the primary function). Unfortunately, the measurements were not performed sufficiently close to the upper asymptote and in such case the underestimation of $K_{c}$ value is impending for the inverse function, cf. $K_{c}=(50.8 \pm$ $\pm 4.2) \mathrm{MPa} \mathrm{m}^{1 / 2}$ with $K_{c}=(60.2 \pm 6.2) \mathrm{MPa} \mathrm{m}^{1 / 2}$ obtained for 
the primary function. On the other hand, the accuracy in $\Delta K_{t h}$ value determination is substantially decreased when the inverse function is used for regression (cf. $\Delta K_{t h}=(5.22 \pm 0.39) \mathrm{MPa} \mathrm{m}^{1 / 2}$ for the inverse function and $\Delta K_{t h}=(5.11 \pm 0.03) \mathrm{MPa} \mathrm{m}^{1 / 2}$ for the primary function in light of standard deviations).

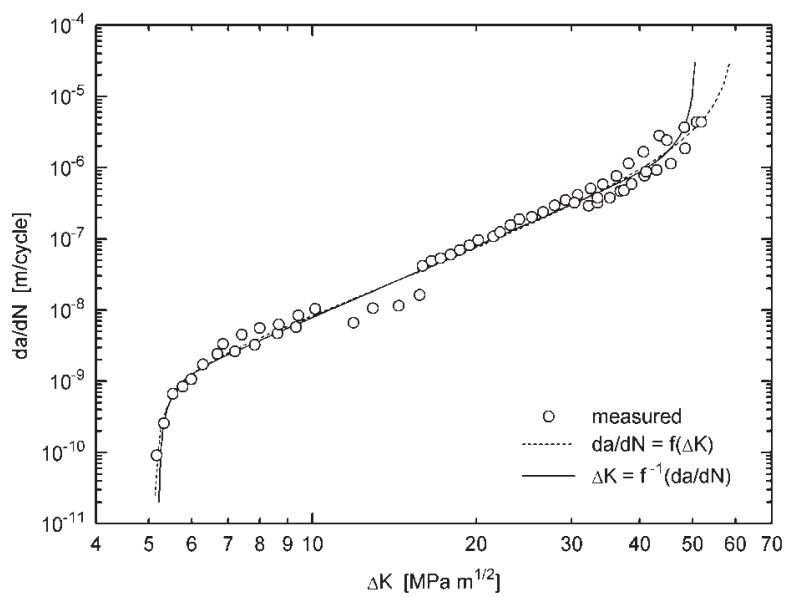

Fig. 4 Fatigue crack growth curves from [1] fitted using primary and inverse function, see Eq. (7)

\section{Discussion}

New equations for fit of fatigue crack growth curves, i.e. Eq (2) for a given loading cycle asymmetry and Eq. (7) for various loading cycle asymmetries, cannot be compared with the NASGRO complex of computer programs, only with the NASGRO equations themselves. With regard to the fitting ability the new equations mostly led to slightly lower sum of squares in comparison with the NASGRO equation, at least in not very large set of growth curves studied up to now. It seems that the new functions can fit better the bends between the Paris straight line and vertical asymptotes. It is probably the reason why the new functions have better extrapolation behaviour.

The new equations can be used also for negative values of loading cycle asymmetry parameter, when the closure of fatigue crack appears. It means that the closure effects are not important for a phenomenological description of fatigue crack growth curves, their role is crucial only when the mechanisms of crack growth at crack closure are studied or modelled. On the other hand, the new equations cannot be probably used when the loaded sample is exposed in aggressive media or when long interruptions of loading are applied, but in such cases also the NASGRO equations can hardly be used universally.

The new and the NASGRO equations describing fatigue crack growth can be compared also from the viewpoint of standard deviations of the regression parameters. The parameter deviations corresponding to the new equations are mostly lower than deviations corresponding to the NASGRO equations. The only exception are parameters $p$ and $q$ describing the bends between the Paris straight line and asymptotes whose deviations are generally high. As an example of comparison the values of slope $n$ of the Paris straight line in Fig. 1 were evaluated as follows: $(3.09 \pm 0.38)$ and $(2.20 \pm$ \pm 0.64 ) for the new and the NASGRO functions, respectively. This slope can also be determined in Fig. 1 graphically (using a tangent to the curve in the point of inflexion) with the result $n \approx 3.1$. It is apparent that $n$ parameter of the new function corresponds very well to the slope of the Paris straight line. Similar conclusion cannot be however drawn for $n$ parameter of the NASGRO function. It seems to be the most important advantage of the new function.

Parameter $C$ in all equations describing the growth rate $d a / d N$ represents an extrapolated value of this rate for $\Delta K=1 \mathrm{MPa} \mathrm{m}^{1 / 2}$ on the Paris straight line characterized by the Paris-Erdogan power law $d a / d N=C \Delta K^{n}$. For most materials the value of $1 \mathrm{MPa} \mathrm{m}^{1 / 2}$ lies rather far from the interval covered by experimental points and therefore parameter $C$ is determined with a high standard deviation. Also in the case of parameter $C$ the standard deviation corresponding to the NASGRO equation is higher than the deviation corresponding to the new equation. The modification of the Paris-Erdogan equation in the form

$$
\frac{d a}{d N}(\Delta K)=C_{0}\left(\frac{\Delta K}{\Delta K_{0}}\right)^{n}
$$

enables a substantial decrease of the standard deviation of parameter $C_{0}$ by a suitable choice of constant $\Delta K_{0}\left(C_{0}=C\right.$ for $\Delta K_{0}=$ $=1 \mathrm{MPa} \mathrm{m}^{1 / 2}$ ) but also illusory correlation between parameters $C$ and $n$ discussed, e.g., in papers $[8,9]$ disappears. For the curve presented in Fig. 1 the lowest variation of parameter $C_{0}$ (i.e. the quotient of standard deviation and average value) $10.21 \%$ was obtained for $\Delta K_{0} \approx 9.68 \mathrm{MPa} \mathrm{m}{ }^{1 / 2}$ in the case of the new function and $22.21 \%$ for $\Delta K_{0} \approx 8.60 \mathrm{MPa} \mathrm{m}{ }^{1 / 2}$ in the case of the NASGRO function. Generally, for usual metal materials the value $\Delta K_{0} \approx 10 \mathrm{MPa} \mathrm{m}{ }^{1 / 2}$ can be recommended. The suitable value of $\Delta K_{0}$ chosen from the interval of stress intensity factor range covered by experimental points decreases not only the variation of parameter $C_{0}$ but also the corresponding covariance coefficients, which simplifies non-linear regression calculations (at least it decreases the number of necessary iterations).

Existence of vertical asymptotes can lead to abnormal fits especially when the dispersion of experimental points closely to the asymptotes is larger. This problem can be solved by exchanging the variables (independent $\longleftrightarrow$ dependent). The inversion function can be explicitly written only for simplified equations; in other cases the numerical calculation of inverse function has to be implemented into a regression procedure using iterative numerical methods, e.g., using the Newton method or the regula falsi method. Derivative of the primary function necessary for the Newton method can be performed analytically (it is rather complicated for Eq. (7)) or calculated numerically when the choice of step length is important.

The application of the inverse function is useful when the dispersion of experimental points close to the asymptotes is large. 
On the other hand, when the bend between the Paris straight line and the $K_{c}(1-R)$ asymptote is not sufficiently covered with experimental points, the $K_{c}$ value determined from regression using the inverse function can underestimate the real $K_{c}$ value. Therefore, the choice between the primary and inverse functions should be performed responsibly and individually in each individual case.

Non-linear regression can be performed also using MS Excel software, which is part of Microsoft Office accessible in the majority of PCs all over the world. For this reason the Solver application is allocated, which contains a very effective minimization procedure giving directly the values of regression parameters. Standard deviations and regression using the numerically calculated inverse function are not directly available but they can be programmed using the Visual Basic implemented in MS Excel.

\section{Conclusions}

1. The new equations (for given loading cycle asymmetry as well as for various asymmetries) allow a slightly better fit of measured fatigue crack growth curves than the corresponding NASGRO equations. They have also better so-called extrapolation behaviour.
2. Parameter $n$ in the new function is numerically equal to the slope of the Paris straight line. It is not valid for parameter $n$ in the NASGRO function.

3. The shift of the curves with changing loading cycle asymmetry can be described by the single parameter $m$ with values from the interval $(0,1)$. This Walker approach is successful for positive as well as for negative values of loading cycle asymmetry parameter $R$ (probably only for non-aggressive media). It means that crack closure effects are not too important for a purely phenomenological description of fatigue crack growth curves.

4. In the case of large dispersion of experimental data close to asymptotes an abnormal fit can be obtained. In this case the exchange of variables can lead to a better fit.

\section{Acknowledgments}

Financial support of the Ministry of Defence of the Czech Republic within the research project MO0FVT0000404 is gratefully acknowledged. Moreover, the authors are grateful to all the authors (see [1, 4-6]) whose original experimental results were used in their considerations and calculations.

\section{References}

[1] FORMAN, R. G., SHIVAKUMAR, V., METTU, S. R., NEWMAN, J. C.: Fatigue Crack Growth Computer Program "NASGRO" Version 3.0. Reference Manual. NASA, Houston 2000 (see also www.nasgro.com).

[2] KOHOUT, J.: International Journal of Fatigue 21, 1999, p. 813.

[3] WALKER, K.: ASTM STP 462, 1970, p. 1.

[4] FORMAN, R.G., HU, T.: ASTM STP 842, 1984, p. 108.

[5] KLESNIL, M., LUKÁŠs, P.: Engineering 22, 1972, p. 269 (in Czech).

[6] KLESNIL, M., LUKÁŠS, P.: Engineering 23, 1973, p. 34 (in Czech).

[7] KLESNIL, M., LUKÁŠ, P.: Engineering Fracture Mechanics 4, 1972, p. 77.

[8] ROMVÁRI, P., TÓTH, L., NAGY, D.: Strength Problems, 1980, o 12, p. 18 (in Russian).

[9] BERGER, F., ZOUHAR, G., TEMPUS, G.: International Journal of Fatigue 23, 2001, p. 383. 